\title{
Transit observation at the observatory in Großschwabhausen: XO-1b and TrES-1
}

\author{
M. Vaňko ${ }^{1}$, S. Raetz ${ }^{1}$, M. Mugrauer ${ }^{1}$, T. O. B. Schmidt ${ }^{1}$, T. Roell ${ }^{1}$, \\ T. Eisenbeiss ${ }^{1}$, M. Hohle ${ }^{1}$, A. Seifahrt ${ }^{1,2}$, A. Koeltzsch ${ }^{1}$, C. Broeg ${ }^{1,3}$, \\ J. Koppenhoefer ${ }^{4}$ and R. Neuhäuser ${ }^{1}$ \\ ${ }^{1}$ Astrophysikalisches Institute und Universitäts-Sternwarte, \\ Schillergäßchen 2-3, 07745 Jena, Germany \\ email: vanko@astro.uni-jena.de \\ ${ }^{2}$ Institut für Astrophysik, Georg-August-Universität, \\ Friedrich-Hund-Platz 1, 37077, Göttingen, Germany \\ ${ }^{3}$ Space Research and Planetary Sciences, Physikalishes Institute, University of Bern, \\ Sidlerstraße 5, 3012 Bern, Switzerland \\ ${ }^{4}$ Max-Planck Institute of Extraterrestrial Physics, \\ Giessenbachstraße, 85748 Garching, Germany
}

\begin{abstract}
We report on observations of transit events of the transiting planets XO-1b and TrES1 with the AIU Jena telescope in Großschwabhausen. Based on our (IR) photometry (in March 2007 ) and available transit timings (SuperWASP, XO and TLC-project-data) we improved the orbital period of XO-1b $(P=3.941497 \pm 0.000006)$ and TrES-1 $(P=3.0300737 \pm 0.000006)$, respectively. The new ephemeris for the both systems are presented.
\end{abstract}

\section{Introduction}

In this paper we present observations of two known transiting planets, $\mathrm{XO}-1 \mathrm{~b}$ and TrES- 1. The observations were carried out with the AIU Jena telescope at the observatory in Großschwabhausen on March 2007. We have used the 25cm Cassegrain telescope installed at the tube of the $0.9 \mathrm{~m}$ telescope. The $R$ and $I$-band images were obtained with the Cassegrain-Telescope-Kamera (CTK) a CCD-camera with 37.7'x37.7' field of view and 1024x1024 pixels. (Mugrauer et al. (2008), in preperation).

For both systems, XO-1b and TrES-1, we have observed one transit event in $I$-band and $R$-band, respectively. The data were reduced by standard IRAF procedures. For data analysis we have used IRAF task chphot, written by $\mathrm{Ch}$. Broeg and based on the standard IRAF routine phot (Broeg et al. 2005). We have then corrected data from systematic effects using the "Sys-Rem" detrending algorithm (Tamuz et al. (2005)) as implemented by Johannes Koppenhoefer. The main aim of our investigation is to improve orbital periods of these transiting planets as well as to discuss possible changes of orbital periods based on their O-C diagrams. For this purpose we collected all available transit times for each transiting system using SuperWASP, XO and TLC-project data.

\section{XO-1b}

We have observed the transit of the exoplanet XO-1b on March 11th 2007. According to the ephemeris provided by McCullough et al. $(2006): T_{\mathrm{c}}(E)=(2453808.9170+E$. $3.941534) d$, this transit corresponds to epoch 92. During observation we have performed $161 \mathrm{I}$-band exposures. Our mean photometric accuracy is 0.008 mag. Due to the variable observational conditions, the photometric accuracy at the beginning of the night was a 
bit worse then at the end. For data analysis we have used aperture photometry on all available images. To get as good as possible transiting light curve, we have used only the most stable comparison stars in the field. After the first run of the artificial-comparisonstar-algorithm we have rejected 168 comparison stars. After repetition of the algorithm
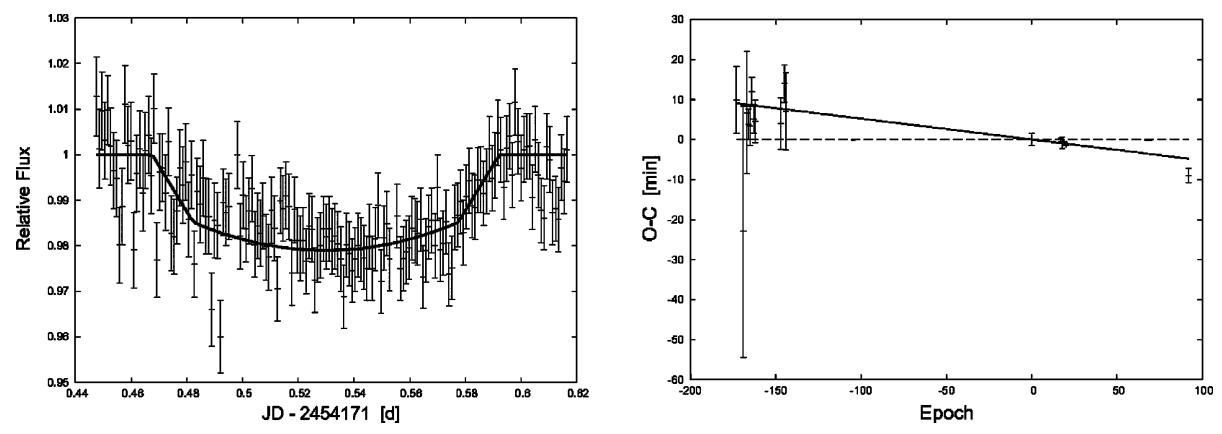

Figure 1. (left plot) Relative $I$-band photometry of XO-1b. (right plot) Transit timing residuals for XO-1b. The dashed line shows the ephemeris given by McCullough et al. (2006). The best fit (dotted line) is representing by the updated ephemeris.

we have rejected other faint stars with low $\mathrm{S} / \mathrm{N}$ and stars suspected of variability. With the remaining 28 most stable stars we calculated the artificial CS. In the resulting light curves we have used Sys-Rem. The algorithm works without any prior knowledge of the effects. The number of effects that should be removed from the light curves is selectable and can be set as a parameter. Using Sys-Rem with two effects the transit itself is disappearing from the light curve. Thus we have used only one effect.

To determine the time of the center transit we have used the fit based on the system parameters of Holman et al. (2006). With the help of the $\chi^{2}$-test, we have determined the time of the midtransit as follows: $T_{\mathrm{c}}(H J D)=(2454171.53188 \pm 0.00130) \mathrm{d}$.

The final time series is plotted in Fig. 1 (left plot). Except of our transit, observed at the observatory in Großschwabhausen, we found 16 other transit times for XO-1b in the literature. We have used the ephemeris of McCullough et al. (2006) to compute "Observed minus Calculated" (O-C) residuals for all 17 transit times. Figure 1 (right plot) shows the differences between the oberved and predicted times of midtransit as a function of epoch. The dashed line represents the ephemeris given by McCullough et al. (2006). We found a negative trend in this (O-C) diagram. For an exact determination of the orbital period we set the transit time with the smallest uncertainties as $T_{0}$. In this case we have used the transit with epoch 20, according to the ephemeris of McCullough et al. (2006). Hence, the transit time for the epoch 0 is $T_{\mathrm{c}}$ (HJD) $=(2453887.74679 \pm$ $0.00015) \mathrm{d}$. Therefore we plotted the midtransit times over the epoch and did a linear fit with fixed $T_{0}$. We got the best $\chi^{2}$ with an orbital period of $P=(3.941497 \pm 0.000006)$ days. Within the error bars almost $70 \%$ of the points are consistent with our calculated period. The remaining measurements are less than 1- $\sigma$ from the new "zero" line (solid line in Fig. 1 - right plot). The resulting ephemeris which is in a good agreement with our observation is: $T_{\mathrm{c}}(H J D)=(2453887.74679+E \cdot 3.941497) \mathrm{d}$.

\section{TrES-1}

The other observation at the observatory in Großschwabhausen was performed on March 15th 2007. We observed one transit of TrES-1 with the same 24.5cm Cassegrain telescope. The light curve consists of $88 R$-band images with 60 s exposures. 

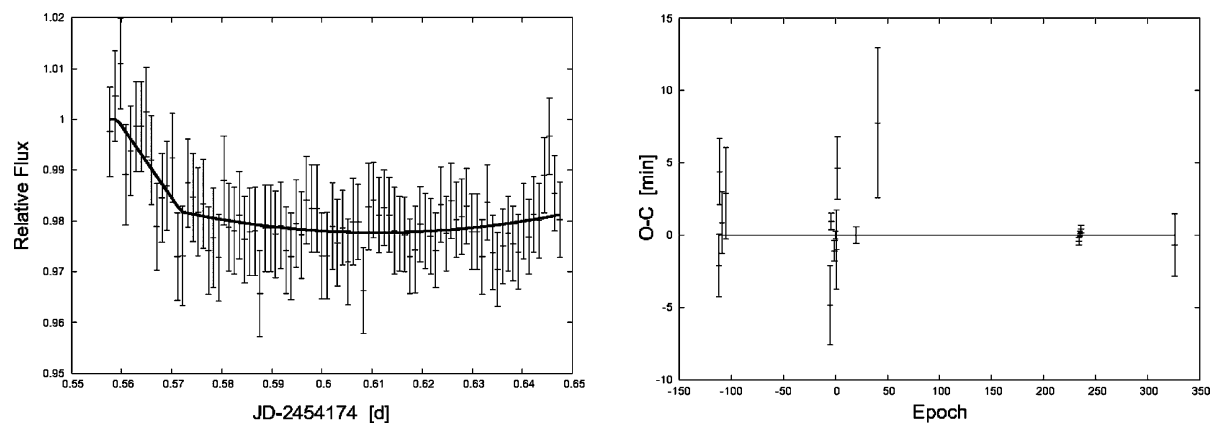

Figure 2. (left plot) Relative $R$-band photometry of TrES-1. (right plot) Transit timing residuals for TrES-1. The dashed line shows the ephemeris given by Winn et al. (2007). The data points are consistent with the constant period.

Unfortunately, our observation was aborted too early and the last part of the light curve is missing. This transit corresponds to epoch 326 of the ephemeris given by Winn et al. $(2007): T_{\mathrm{c}}(E)=(2453186.80603+E \cdot 3.0300737) \mathrm{d}$. In this case our mean photometric accuracy is 0.009 mag. The data reduction and analysis was carried out in the same way as in the case of XO-1b. For calculation of the artificial comparison stars, we have used 34 most stable stars with a good S/N. As we did not have light from out of transit which allow us to find the systematic effect, the Sys-Rem was not used. The determination of the transit center was done in the same way as in the case of XO-1b. After normalization we did a fit of the light curve using the system parameters by Winn et al. (2007). Using the theoretical light curve and the $\chi^{2}$-test, it was possible to estimate the center of the transit even without the egress. The minimal value of $\chi^{2}$ corresponds with the following midtransit time: $T_{\mathrm{c}}(H J D)=(2454174.60958 \pm 0.00150) \mathrm{d}$.

The resulting light curve is shown in the Fig. 3 (left plot). For TrES-1 we found 15 midtransit times in the literature. The transit of epoch 40.5 (forced $e=0$ ) according to the ephemeris given by Winn et al. (2007) is even a secondary transit observed by Charbonneau et al. (2005). With all 16 available transit times we determined the transit and secondary-eclipse timing residuals for TrES-1. The calculated times (using the ephemeris of Winn et al. (2007)) have been subtracted from the observed times. The resulting (O-C) diagram is shown in the Fig. 2 (right plot). The point lie on the horizontal line. It means that data are consistent with a constant period which confirmes the ephemeris given by Winn et al. (2007).

\section{References}

Broeg, Ch., Fernndez, M., \& Neuhäuser, R. 2005, AN, 326, 134

Charbonneau, D., et al. 2005, ApJ, 626, 523

Holman, M. J., et al. 2007 ApJ, 664, 1185

McCullough, P. R., et al. 2008, ApJ, 648, 1228

Mugrauer, M., et al. 2008, AN, in preperation

Tamuz, O., Mazeh, T., \& Zucker, S. 2005, MNRAS, 356, 1466

Winn, J. N., Holman, M. J., \& Roussanova, A. 2007, ApJ, 657, 1098 